\title{
DNA discourse
} Molecular biology is the subject and the objects of attention include a gel electrophoresis comet assay, an
adenovirus expression kit, proof-reading polymerases, and a large-scale mouse cDNA expression array.

\section{SFM 25}

Kontron Instruments

A spectrofluorometry instrument with WIND 25 software

Using Microsoft Windows as its platform, WIND 25 is a 32-bit application that allows fast calculations and multitasking operations. It is designed for user friendliness, with a graphical interface that guides the user through all the functions and features of the system. Advanced calculations offered with this system include enzyme determination and substrate, Michaelis-Menten, ratio and purity calculations. The instrument uses a high-intensity xenon light source, a double-beam optical system with blazed gratings and a high-sensitivity photomultiplier, to provide higher sensitivity and consistent, reproducible results.

Reader Enquiry No. 100

\section{Micro Imager}

From Bio Image

\section{Fast tritium detection in ISH experiments}

Developed by Biospace Mesures in France, this instrument detects tritium or other $\beta$-emitting isotopes in tissue sections and in situ hybridization (ISH) experiments up to $\times 50$ faster than film, says Bio Image. The system achieves a spatial resolution of $15 \mu \mathrm{m}$ and is also capable of dual-isotope detection. It can simultaneously detect tritium and another isotope in the same sample, thus eliminating the error when overlaying two samples. The system is used for $\beta$ detection in applications, such as ISH experiments, immunocytochemistry, receptor-ligand binding assays and metabolic tracer experiments. The Micro Imager detects all $\beta$-emitting isotopes with a detection efficiency of 60-90 per cent and background noise less than 0.01 c.p.m. $\mathrm{mm}^{-2}$. Real-time imaging allows the user to stop the acquisition at any time, reducing the risk of overexposure or under-exposure.

Reader Enquiry No. 101

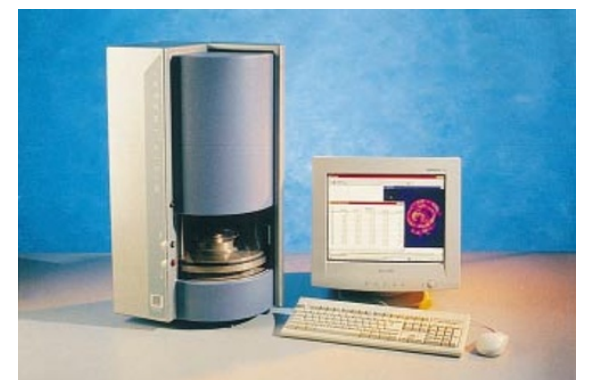

Bio Image's fast tritium detection system.

\section{Cometslide}

From Trevigen

A single-cell gel electrophoresis (comet) assay

The assay kit allows the quantitative evaluation of DNA fragmentation associated with DNA damage or apoptosis. It provides low melting point (LMP) agarose, lysing buffer, nucleic acid stain and the company's CometSlide - a specially treated microscope slide, which allows direct application of cells in LMP agarose to the surface, without pre-treatment. The CometSlide features a specially designed hydrophobic barrier for incubating cell samples with Trevigen's DNA repair enzymes, allowing analysis of specific types of gene damage.

Reader Enquiry No. 102

\section{GS2000}

From UVP

A DNA fragment analyser for automated fluorescence, sizing, sequencing and quantification

This system utilizes an ultra-thin gel apparatus and is stated to have the resolution of single-channel capillary systems, but with the advantage of being able to run many samples in parallel. Gels are poured in situ and can be re-used up to five times. Samples from 50-100 $\mu$ l can be loaded with standard pipettors using 48- and 64-well combs. Real-time electrophoresis detection will analyse fluorescently labelled DNA as it migrates through the gel. The sensitivity and resolution of the system is said to allow the system to detect and size 100 attomoles of DNA in a single peak. The system is said to cost less to operate, owing to reagents savings. It will detect most commercially available fluorphores. For non-denaturing applications ethidium bromide can be used to detect as little as 500 attomoles of DNA.

Reader Enquiry No. 103

\section{Mirus' Labellt}

From Pan Vera

Kits for efficient one-step, non-radioactive

labelling of DNA or RNA with a single reagent

This method is said to tag covalently and non-destructively original substrate nucleic acids. In contrast to some laborious and poorly controlled enzymatic reactions, these reactions are said to be simple, non-enzymatic and result in reproducible products and yields. In addition, the reactions can be scaled in a single tube without loss of efficiency. Products can be used in many molecular biology applications, including FISH and

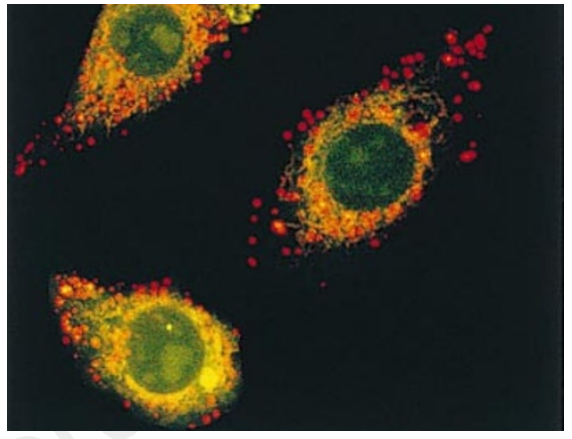

Label It: non-radioactive labelling from Pan Vera.

DNA or RNA transport studies. The kits are available for biotin, digoxin, rhodamine and fluorescein labelling applications.

Reader Enquiry No. 104

\section{Adenovirus expression vector kit \\ From TaKaRa \\ A more efficient means of generating recombinant adenovirus for gene transfer that is based on the COS-TPC method}

Adenovirus vectors have not been widely used for gene transfer because the procedure for generating adenovirus vectors is laborious and inefficient. The COS-TPC method utilizes genomic DNA-terminal protein complex (DNA-TPC) and a cosmid with the target gene inserted. Whereas, conventional methodology uses a plasmid and adenovirus genomic DNA without terminal protein (TP), though both terminals of the adenovirus genomic DNA are originally tagged with TP. According to the manufacturer, high-titre virus can be obtained using the COS-TPC method $\left(10^{8}-10^{9}\right.$ virus per millilitre), which can be further concentrated to $10^{11} \mathrm{pfu} \mathrm{ml} \mathrm{m}^{-1}$. This is said to result in a system that can transfer the target gene to nearly 100 per cent of adherent cells. It is available for a wide range of animals and for infecting both resting cells and proliferating cells. It can be targeted to a number of differentiated and undifferentiated cultured animal cells, including nervous system cells. Reader Enquiry No. 105

\section{Imagene}

From Life Science Resources

An image analysis and processing platform available for gene imaging and other molecular biology applications

This platform uses the latest fluorescence probes, such as green fluorescent protein (GFP) and chemiluminescent probes (aequorin and luciferace), to provide an integrated system for the study of gene 
expression in living and dead cells. Imagene can acquire either single or time-lapse images labelled with one or more fluorescent probes, specific to the gene or genes of interest. Image analysis tools provide image montages, intensity plots with time graphical overlays that relate fluorescence to gene activity. The system is fully integrated with the company's 16-bit digital cameras available with resolution up to $1,317 \times 1,035$ pixels. Back-thinned CCDs are available for studies utilizing the low-light emitting chemiluminescent probes, aequorin and luciferase. Automated control of external hardware is provided as an integral part of the system, including a high-speed filter wheel, a monochromator, $z$-stepper stage control and camera shutter. The image processing digital deconvolution software is also available as an optional upgrade to the system. This image enhancement capability can smooth, sharpen or remove out-offocus haze from two- or three-dimensional microscope images.

Reader Enquiry No. 106

\section{TGGE}

From Biometra

Rapid mutation detection by temperaturegradient gel electrophoresis (TGGE)

According to the manufacturer, this new instrument is useful for both mutant screening and allele typing. It uses a specially designed electrophoresis unit that utilizes Peltier elements for precise control of the chamber temperature. In addition, a combined system controller and power supply provides the run conditions for gradient or standard temperature profiles, which is necessary for DNA migration. TGGE is said to be an improvement over other DNA screening methods in that it can detect almost 100 per cent of all mutations, says Biometra. Reader Enquiry No. 107

\section{GDS-20}

From ATR

A gel drying system that provides efficient oilfree drying at an economical price

The system incorporates the HGD-10 largeslab gel dryer, the GDTT inlet/outlet trap system, a TD-20 diaphragm pump, and all the connections and tubing required for assembly. Solid, Teflon pump heads, continuous moulded diaphragms and a multiport valve design are said to provide greater reliability than other diaphragm pumps. Unlike water aspirators, diaphragm pumps maintain a constant vacuum, resulting in more consistent gel drying. The expansion flask and charcoal filter provide a vapourfree, odour-free environment and isolate radioactive waste. (Charcoal filters are inexpensive and easy to replace.) Designed for convenience, as well as performance, the

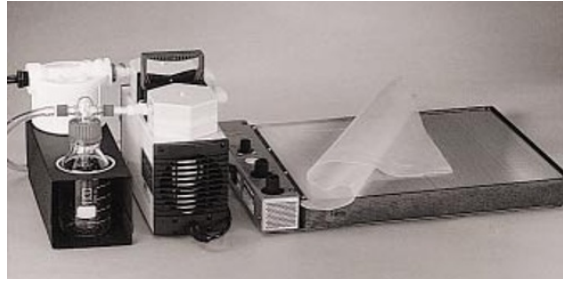

ATP offers its GDS-20 gel drying system.

system can be installed and ready for use in minutes, says ATR.

Reader Enquiry No. 108

\section{GeneGenius}

From Syngene

A complete gel documentation and analysis system for molecular biology

GeneGenius is a PC-based configuration with a darkroom, camera and transilluminator, as well as the GeneSnap acquisition system, GeneTools one-dimensional analysis software and a thermal printer. These modules provide image acquisition and processing for laboratories that are working with DNA and protein gels, autoradiographs and other types of gel media. The system combines a simple user interface and is supplied with a PC that has a CD-ROM drive, keyboard, mouse and a Windows 95 or Windows NT user interface. Included with this package are word-processing, spreadsheet and publishing programs so that results and data can be exported for publication or analysis. GeneTools analysis software is also available as a stand-alone package for use with images captured using different systems. It handles a wide range of molecular biology applications: agarose gels, Southern blots, western blots, northern blots, autorads and SDSPAGE. An ELISA plate/dot-blot and colony counting options are also available. GeneGenius is also said to perform band quantification, molecular size determination, band matching, band comparison, volume measurements, $\mathrm{R}_{\mathrm{F}}$ determination, dendograms and profiling comparisons.

Reader Enquiry No. 109

\section{6-well gel electrophoresis}

From MadgeBio

Using Madge gels, this system is designed for molecular biology reactions in 96-well plates

Developed in the UK, this system utilizes existing gel electrophoresis tanks and is said to reduce labelling confusion and transfer times from the reaction plate to the gel. High throughput can be achieved in a shoebox-sized gel tank, processing up to several thousand samples in a few hours, says MadgeBio. High resolution is achieved in the 20-1,000-bp range. Moreover, automation is now possible and high-quality analytical software is available to analyse the resulting gels. The gel-plate measures
$170 \mathrm{~mm} \times 110 \mathrm{~mm}$ and fits into conventional gel electrophoresis tanks with $968-\mu l$ wells. The gel is set at an angle to the field, and provides a $2.6-\mathrm{cm}$ separation run adequate separation for most applications. The kit provides the means to produce either agarose or acrylamide gels.

Reader Enquiry No. 110

\section{FTA Gene Guard}

From Life Technologies

For collection, storage and purification of blood samples for genetic analysis

This technique allows simple collection and long-term, room-temperature storage of blood samples for human identity testing and genetic analysis. The FTA-treated matrix protects DNA during storage and allows simple purification of high-quality genomic DNA. Matrix-bound DNA enables consistent PCR and RFLP results, and is said to be well suited for high-throughput applications. The FTA card consists of filter paper impregnated with a proprietary formulation of denaturants, which are designed to maintain the integrity of the DNA contained in the specimen, and to prevent growth of bacteria and microorganisms. DNA can be isolated from blood (up to about $500 \mu \mathrm{l}$ ), buccal swabs, epithelial cells, bacteria, viruses and cultured cells. Once biological fluid is spotted on the FTA card, DNA purification takes less than an hour, states the manufacturer. Blood spotted on the card does not require refrigeration, and can be stored indefinitely at room temperature in a typical filing system, eliminating the need for large freezers.

\section{Reader Enquiry No. 111}

\section{Reagents}

\section{India-HRP}

From Pierce

A new probe, together with its metal-activated derivatives for the detection of target molecules

This derivative of horseradish peroxidase allows metals to be bound in an active form for subsequent interaction and detection of target molecules. The active chelator has similar binding capabilities to those reported for iminodiacetic acid. The India HisProbe-HRP, a nickel-activated derivative of HRP, has been optimized for direct detection of recombinant histidine-tagged proteins and other histidine-rich proteins. India PhosphoProbeHRP, an iron-activated derivative of HRP, is phosphate $\left(\mathrm{R}-\mathrm{PO}_{3}\right)$-specific when coupled with a novel treatment termed 'reactive chemical binding' (RCB). This probe has been optimized for direct detection of phosphoester $\left(\mathrm{R}-\mathrm{PO}_{3}\right)$ molecules, such as nucleotides or protein/peptides containing phosphoserine, phosphothreonine and phosphotyrosine.

Reader Enquiry No. 112 


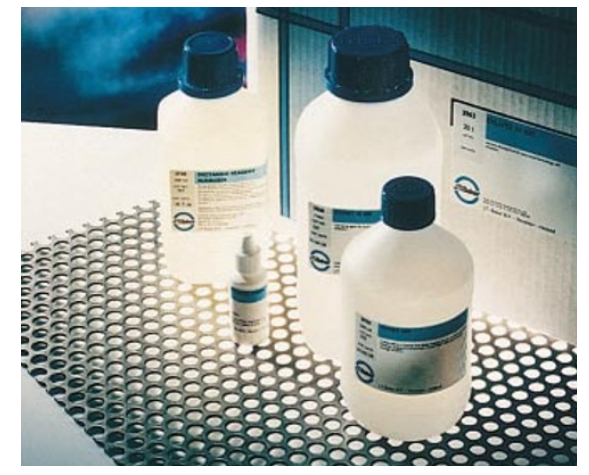

Hyped on hypochlorite solution for cell analysers.

\section{Hypo Chlorite}

From Mallinckrodt Baker

An improved hypochlorite reagent that may mean longer life, simpler cleaning procedures and reduced down-time for cell analysers

By adding special tube-protecting compounds to its traditional product, the manufacturer may help to ensure users of continued malleability of plastic tubes and components together with suppressed noise. This new, odourless hypochlorite not only offers more effective cleaning, according to the manufacturer, but is considerably less corrosive than alternative products.

Reader Enquiry No. 113

\section{Prime RNase inhibitor}

From Flowgen

A human placental inhibitor that is said to reduce costs by 50 per cent

According to the manufacturer, this is a broad specificity, protein-based RNase inhibitor that is 50 times more active at inhibiting RNase A, B and C than most human placental inhibitors. It is said to exhibit activity and stability over a broad range of DTT concentrations, temperature and $\mathrm{pH}(5.0-9.0)$. It is stable at elevated temperatures $\left(37^{\circ} \mathrm{C}\right)$ for as long as two hours. Moreover, this inhibitor is suitable for protecting RNA samples from degradation in CDNA synthesis; in vitro transcription using the SP6, T7 and HeLa cell

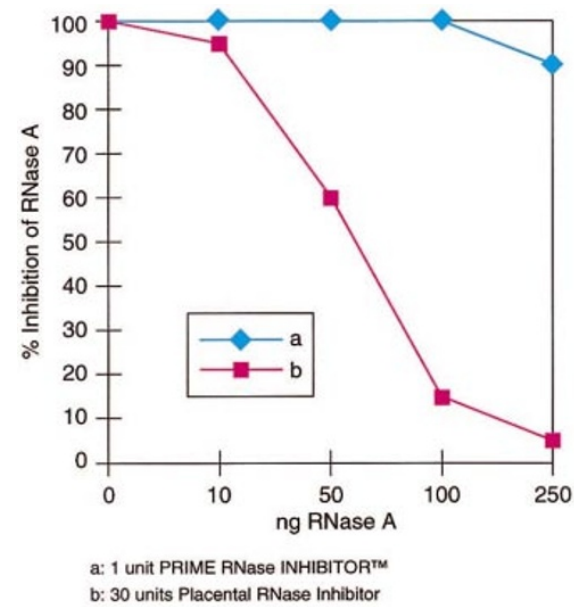

Potency of Prime RNase inhibitor from Flowgen. extract systems; in in vitro translations that use rabbit reticulocyte lysates and wheat germ extracts. Additionally, Prime RNase inhibitor can prevent RNA degradation in cell extracts prepared under non-denaturing conditions. It can be utilized in first-strand synthesis before PCR and in the RT-PCR of RNA isolated from single cells. This reagent should also find use in gel retardation assays, the isolation of an RNA binding protein by oligonucleotide affinity purification and in nuclear run-on transcription assays.

Reader Enquiry No. 114

\section{Complementary tools}

Dynabeads Oligo $(\mathbf{d T})_{25}$

From Dynal

Beads for mRNA isolation and subsequent production of subtracted $c D N A$ probes

This method can be used for the screening and isolation of rare, differentially expressed mRNAs. The first step is the isolation of polyA+ RNA, using Dynabeads Oligo (dT)25. Then, instead of eluting the captured mRNA, a solid-phase cDNA library is constructed directly on the bead's surface, using the oligo(dT) sequence bound to the bead as a primer. This library is specific to a particular cell type or tissue. Next, subtracted first-strand cDNA is immobilized on beads and this is hybridized with mRNA from the target material, leaving subtracted mRNA in the supernatant after the removal of common mRNA captured with the Dynabeads. Unique mRNA is subsequently reverse transcribed to radiolabelled cDNAs and used to screen cDNA libraries. In addition, both first- and second-strand cDNA are synthesized for cDNA cloning. Magnetic handling is said to minimize losses at each step, and also enable simple and rapid buffer changes to optimize conditions for hybridization and specific enzymatic reactions. The subtracted cDNA bound to the Dynabeads can be stored and reused to screen multiple libraries.

Reader Enquiry No. 115

\section{Atlas}

From Clontech

A large-scale mouse cDNA expression array for throughput monitoring of gene expression

The array includes 588 genes involved in key processes, such as oncogenesis, growth and cell-cycle control, apoptosis, embryogenesis and signal transduction. Two arrays are included in each order to facilitate differential expression profiling, for instance, to compare expression levels in transgenic knockout mice to those in normal mice. Because no special equipment is required for analysis, the Atlas mouse array is an economical way to explore the relationships among genes. Arrays are provided on nylon membranes that can be hybridized
ADVERTISEMENTS

DNA SEQUENCING

OLIGONUCLEOTIDES

GENOTYPING

By research labs - For research labs

Call us for more information

Molecular Biology Services

King's College School of Medicine and Dentistry

Tel: +441713463126

Fax: +44171733 3877

www.kcl.ac.uk/links/mbs

READER ENQUIRY NO. 9

NEWCASTLE UNIVERSITY MOLECULAR BIOLOGY UNIT

\section{PROTEIN SEQUENCING}

Membrane-bound, solid or solution samples $£ 12$ per cycle

5 cycles minimum - no set up charge

For further information contact Dr. Joe Gray

Phone: +441912228612

Fax: $\quad+441912228129$

Email: JOE.GRAY@newcastle.ac.uk

READER ENQUIRY NO. 4

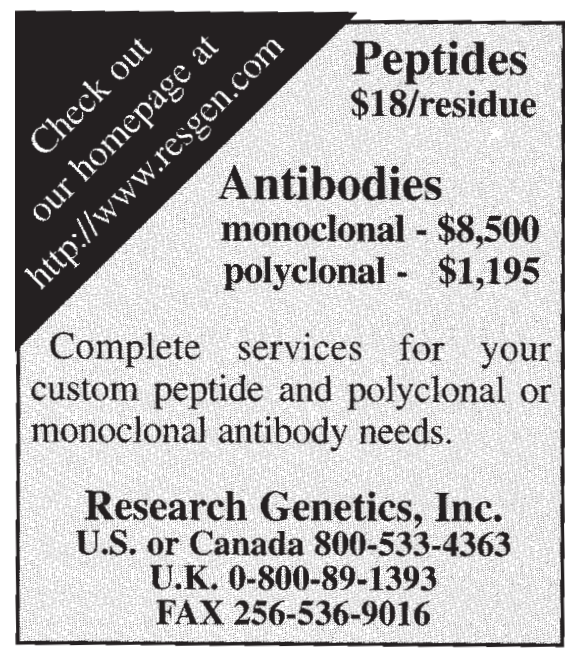

READER ENQUIRY NO. 29

with a ${ }^{32}$ P-labelled first-strand cDNA probe and visualized by autoradiography or phosphorimaging.

Reader Enquiry No. 116

\section{Polymerases and priming}

HS TaQuant-Off

From Quantum Biotechnologies

A reagent that blocks thermostable DNA polymerase activity during PCR set-up

This compound can be used with various 
native or recombinant enzymes, and its inhibiting activity is completely reversed above $48{ }^{\circ} \mathrm{C}$. According to the manufacturer, pre-mixing solutions of Taq DNA polymerase and HS TaQuant-Off do not lead to loss of polymerase activity even after several hours at the room temperature. The company also supplies various thermostable DNA polymerases that are licensed by Hoffman-La Roche for PCR and RT-PCR. Native Taq polymerase is a highly purified $94 \mathrm{~K}$ enzyme isolated from Thermus aquaticus. BioTaq is a recombinant enzyme obtained by over expression of the T. aquaticus polymerase gene in Escherichia coli. Q-BioTaq polymerase is a truncated form of the enzyme, missing the $5^{\prime} \rightarrow 3^{\prime}$ exonuclease activity. It provides enhanced thermostability up to $98^{\circ} \mathrm{C}$ and works over a broad range of conditions, facilitating PCR protocol optimization. The low error rate of this enzyme makes it useful for multiplex amplifications, RAPDs and sequencing procedures. The BI-TAQ DNA polymerase is purified from Thermus brokianus and is used for generating long amplicons (up to $6 \mathrm{~kb}$ ) without a complex and time consuming PCR optimization step. This enzyme is suitable for generating PCR products from GC-rich templates and is said to be preferable to native Taq polymerases for sequencing applications as it has a twofold lower error rate.

Reader Enquiry No. 117

\section{PuodNA polymerase}

From Boehringer Mannheim

A new proof-reading thermostable

DNA polymerase

The company claims that this new enzyme product has a lower error rate than any other available on the market, making it well suited to high-fidelity PCR. It amplifies blunt-ended fragments up to a stated $2.9 \mathrm{~kb}$ and can therefore be used directly for bluntend ligation without any pretreatment of the ends, says Boehringer. It exhibits increased thermal stability with a half life of more than two hours at $100{ }^{\circ} \mathrm{C}$. The inherent $3^{\prime} \rightarrow 5^{\prime}$ exonuclease proof-reading activity of $P w o$ results in a stated tenfold increase in fidelity of DNA synthesis compared to Taq. The enzyme was originally isolated from the archaebacterium Pyrococcus woesei. Boehringer Mannheim supplies the recombinant form cloned in Escherichia coli and purified to be free of unspecific endoor exonucleases.

Reader Enquiry No. 118

\section{Primer Premier Mini \\ From Premier Biosoft \\ A free program that analyses oligonucleotides to be used in PCR, sequencing or hybridization}

With this program users simply type in the sequence of an oligo, and with a single mouse click, the program calculates the melting temperature using nearest neighbour theory, GC percentage, free energy $(\Delta \mathrm{G})$ in kcals $\mathrm{mol}^{-1}$, optical activity and degeneracy. In addition, all secondary structures that reduce priming efficiency, such as hairpins and dimers, are shown ranked by structural stability. A primer database makes it easy to store the names and sequences and frequently used primers. The program can be downloaded free from the company's website at $<$ www.PremierBiosoft.com $>$.

Reader Enquiry No. 119

These notes are compiled by Brendan Horton from information provided by the manufacturers. For more details, fill in the reader service card bound inside the journal.

\section{Senthing for Something? Seariching for Something?}

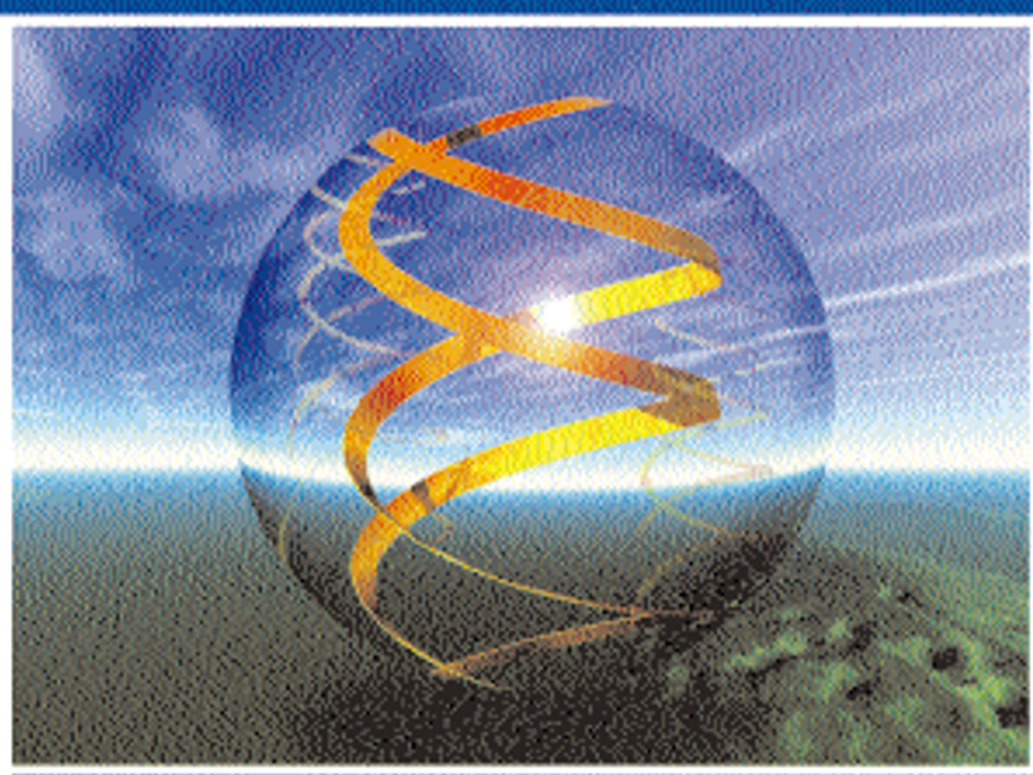

\begin{tabular}{|c|c|}
\hline DMIGA 1.J far V/indav & \\
\hline 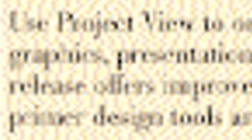 & 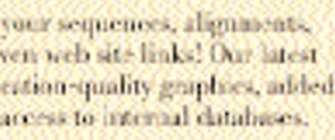 \\
\hline Necvecto 60 bo 1 & \\
\hline 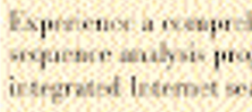 & 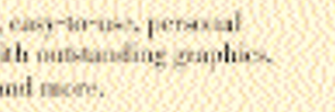 \\
\hline GCG's Wisconsia Padk & rUXXX and Open VMS \\
\hline 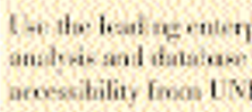 & 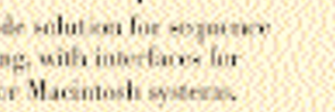 \\
\hline SPS' poelaces for SIV & \&_Mated and Ptrap \\
\hline 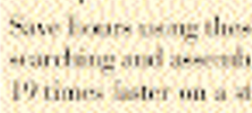 & 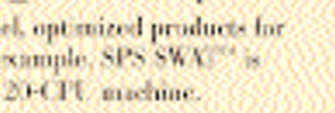 \\
\hline $\begin{array}{l}\text { Call us ar wisit cur ve } \\
\text { hittp:/ umwoxmal. }\end{array}$ & $\begin{array}{l}\text { bar search is cuet! } \\
\text { dohio }\end{array}$ \\
\hline & \\
\hline $\begin{array}{l}\text { Eurepe } \\
\text { tox: }+441865784603 \\
1865784501\end{array}$ & $\begin{array}{l}\text { Worldwide } \\
\text { hitp://ww..cxnol.con/ } \\
\text { 1-403-879-5300 }\end{array}$ \\
\hline
\end{tabular}

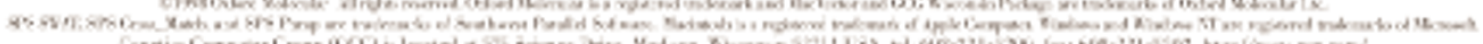

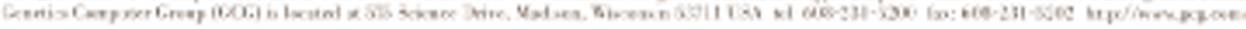

